\title{
An association between phantom limb sensations and stump skin conductance during transcutaneous electrical nerve stimulation (TENS) applied to the contralateral leg: a case study
}

\author{
Joel Katz, Christopher France and Ronald Melzack
}

\begin{abstract}
Sumnary This report describes a placebo-controlled study of transcutaneous electrical nerve stimulation (TENS) applied to the contralateral lower leg and outer ears of an amputee with non-painful phantom sensations. The subject received TENS or placebo stimulation on separate sessions in which baseline periods of no stimulation alternated with periods of TENS (or placebo). Throughout the two sessions, continuous measures of stump skin conductance, surface skin temperature and phantom intensity were obtained. The results showed that TENS applied to the contralateral leg was significantly more effective than a placebo in decreasing the intensity of phantom sensations, whereas stimulation of the outer ears led to a non-significant increase. The pattern of electrodermal activity on the TENS session was consistently linear during baseline periods, indicating a progressive increase in sympathetic sudomotor activity. In contrast, during periods of electrical stimulation the pattern of electrodermal activity was consistently curvilinear indicating an initial decrease followed by an increase in sudomotor responses. Changes in stump skin conductance correlated significantly with changes in phantom sensations both in TENS and placebo sessions suggesting a relationship between sympathetic activity at the stump and paresthesias referred to the phantom. Two hypotheses are presented to account for these findings.
\end{abstract}

Key work: Phantom limb pain; Electrodermal activity; Sympathetic nervous system; TENS

\section{Introduction}

A number of case reports have recently shown that transcutaneous electrical nerve stimulation (TENS) or local anesthetic agents applied to the contralateral limb can be effective in reducing phantom limb pain $[5,11,22]$. However, to our knowledge there are no reports of the effect of TENS applied to the contralateral limb in amputees with 'normal' non-painful phantoms.

Wall [21] has proposed that a normal phantom occurs whenever nerve impulses from the periph- ery are blocked or otherwise removed. This lack of normal input releases spinal cord cells subserving the limb from inhibitory control, and in the case of amputation, results in non-painful paresthesias of striking clarity referred to the lost part. With the passage of time there is a gradual fading or suppression of the phantom. However, the application of TENS directly to the stump can sometimes exaggerate a fading phantom or even revive one that has been suppressed.

This report describes a placebo-controlled single-case study of TENS applied to the contralateral leg and outer ears of an amputee reporting a non-painful phantom limb. The object of the study is to examine whether (1) changes in phantom intensity reported during TENS result from 
its physiological actions or are attributable to the demand characteristics of the situation, expectancy for change, and various experimenter qualities, (2) changes in phantom intensity are limited to contralateral limb stimulation or can be brought about by stimulation of another distant body site, and (3) alterations in electrodermal activity or skin temperature are associated with changes in the intensity of phantom sensations.

\section{Subject and methods}

\section{Case E20}

The subject was a 23-year-old man who had lost his right leg above the knee as the result of a motorcycle accident in early September 1987 . He was initially seen as part of a larger study on phantom limb pain 61 days after the amputation while a patient at a Montreal area hospital, where he had been transferred for prosthetic fitting and training. At the time of the initial interview he reported several distinctly different types of phantom sensation which occurred with varying regularity.

\section{Phantom limb pain}

Immediately following the amputation he felt painful paresthesias in the phantom foot. Several days postoperatively he began to develop paroxysmal 'spasms' in the phantom calf, knee, and thigh as though suddenly 'stabbed with a sharp pencil.' These pains occurred without warning and at times repeatedly, with successive 'stabs' separated by several seconds.

\section{Somatosensory memories}

On a number of occasions the subject had noticed painful and non-painful phantom sensations that were replicas of experiences felt in the right leg prior to amputation. He reported feeling what he termed 'growing pains' on the anterior aspect of his phantom shin. These were in all respects the same pains which he had originally experienced as an adolescent approximately 8 years earlier but had not felt in the intervening years until the amputation.

Two sensations which he originally experienced at the accident scene have recurred as phantom phenomena. He described the first as the beginning of a muscle cramp in his right calf that threatened repeatedly to develop into a full-blown spasm but never did. The second occurred a short time later when the ambulance attendants arrived and were required to cut off his right boot in order to release his foot. He felt a sensation of 'cool fresh air' on his foot and a considerable release of constrictive pressure as it was freed from the boot. The phantom sensation, which has recurred a number of times, consists of the latter sensation and does not include the 'cool fresh air' component. This phenomenon of past somatosensory events re-experienced in the phantom is a frequent sequela of amputation and has been recently described elsewhere [13].

At the time of the initial interview, he reported that the 'spasms' had decreased in frequency, occurring approximately once a day for several seconds. The painful paresthesias defined a region of his phantom foot that included the instep, toes, and occasionally the ankle. They had decreased in intensity but were constantly present and still painful. He felt that his phantom leg had 'telescoped' slightly, estimating it to be 1-2" shorter than his left leg. It was flexed at the knee in a fixed position whenever he was not wearing his prosthesis and otherwise assumed the postures of the artificial limb. He frequently experienced the illusion that the phantom leg penetrated solid objects such as the mattress when he lay supine in bed. or tabletops upon which he often rested his stump.

The subject had participated in an earlier study (unpublished) in which he had received electrical stimulation applied at his outer ears on one session and placebo on a control session. The results from both sessions showed statistically significant correlations between skin conductance at the stump, at the contralateral limb, and phantom pain intensity. The subject had also received several treatments of TENS applied to the contralateral leg when he was in the hospital which had led to a reduction in the painful paresthesias. On the basis of these findings we decided to examine the effects of TENS applied to the contralateral leg and outer ears on stump skin conductance, temperature and phantom limb sensa- 
tions. The present set of results was obtained 138 days post amputation. By then the painful spasms had become less frequent. The paresthesias persisted at a less intense level which he considered annoying but no longer painful. They defined a smaller region of the phantom limb which included the outer 3 toes.

\section{Experimental apparatus}

Transcutaneous electrical nerve stimulation (TENS) was applied to the contralateral leg and outer ears using a 2-channel Grass model S88 stimulator. Two Grass model SIU5 stimulus isolation units were used to isolate the subject from the stimulator. Bilateral ear stimulation (BES) was delivered through 2 silver earrings which gently clasped the subject's earlobes. Contralateral leg stimulation (CLS) was delivered using 2 standard rubber TENS electrodes (Agar Electronics). Stump skin conductance (SC) measurements were obtained using a portable Thought Technology biofeedback module with digital display (SC200T) and $\mathrm{Ag} / \mathrm{AgCl}$ Beckman electrodes. The electrode paste consisted of a mixture of physiological saline and a neutral ointment cream having the recommended concentration of approximately $0.05 \mathrm{M}$ $\mathrm{NaCl}$ [10]. Surface skin temperature (ST) measurements at the stump were obtained using a Yellow Springs Instruments (YSI) digital thermometer, Model 49TA, and a YSI Model 409A temperature probe.

The subject rated changes in the perceived intensity of phantom limb sensations (PLS) by turning a dial which allowed $180^{\circ}$ of rotation. The $90^{\circ}$ setting was labeled 'usual,' $0^{\circ}$, 'less than usual,' and $180^{\circ}$ 'more than usual.' 'The dial was connected to a $1.35 \mathrm{~V}$ mercury battery via a $10,000 \Omega$ potentiometer and the output fed into a digital volt meter which registered 0 through 0.675 to $1.35 \mathrm{~V}$ corresponding to the $0^{\circ}, 90^{\circ}$, and $180^{\circ}$ settings, respectively. Measurements were obtained on a continuous basis and digital displays, including a digital timer, were videotaped for later scoring.

\section{Procedure}

The subject was tested on 2 consecutive evenings. When he arrived at the laboratory the sub- ject was seated in a quiet room where the experimental procedures were explained to him and his informed consent was obtained. He removed his prosthesis and sat quietly for $20 \mathrm{~min}$ to allow the stump to adjust to the laboratory environment. Skin conductance electrodes and temperature probe were then placed on the distal portion of the stump approximately $5 \mathrm{~cm}$ from its end. Earring electrodes were placed in position and TENS electrodes were placed on the subject's lower left leg (over the soleus and gastrocnemius muscles). The subject was instructed in the use of the dial to monitor phantom limb sensations. He was told to pay close attention to his phantom limb and to turn the dial in the appropriate direction if he noticed any type of change in sensation. He was to indicate the magnitude of a change by the size of the angle of arc produced by turning the dial. Recording of physiological measures was initiated when the subject understood how to use the dial and had begun monitoring his phantom limb.

\section{Design}

Each session was divided into 7 consecutive periods of 9 min each, including an initial resting baseline (B1), contralateral leg stimulation (CLS), a recovery baseline (B2), bilateral ear stimulation (BES), a second recovery baseline (B3), combined CLS and BES (CLS/BES), and a final resting baseline (B4).

The subject received placebo stimulation on the first session and TENS on the second. The procedure on both sessions was identical except that on the placebo session, non-conducting leads connected the stimulator and isolation units so that the subject received no current. At the beginning of each stimulation period on both sessions the experimenter turned on the Grass stimulator which was within view of the subject. He was told that he might or might not feel the effects of stimulation and that, when he did, he was to inform the experimenter. During stimulation periods of the TENS session, stimulation intensity was increased until the subject reported a strong but not uncomfortable sensation. Small muscle contractions at the stimulation sites on the left lower leg were visible during CLS and CLS/BES. During 'stimulation' periods of the placebo session the experi- 
menter slowly turned the dial that controlled the intensity, asking the subject to report if and when he felt the stimulation. After some time the experimenter stated that the current was at the appropriate level. On both sessions the subject was periodically asked if he could feel something and to indicate if it became too strong.

TENS at the outer ears was delivered, using the same procedure, at $4 \mathrm{~Hz}, 125 \mathrm{msec}$ pulse duration and an intensity ranging between 20 and $24 \mathrm{~V}$. For comparative purposes we decided to use the same parameters for BES as we had in our previous work $[12,15]$. CLS was delivered at $100 \mathrm{~Hz}, 80$ $\mu \mathrm{sec}$ pulse duration and an intensity ranging between 100 and $120 \mathrm{~V}$. These were the stimulation parameters which the subject had received during his previous course of contralateral TENS.

\section{Physiological data reduction}

Physiological data collected during each session were reduced in the following manner. The videotapes were reviewed and recorded values of SC,
ST, and PLS were obtained at 2 sec intervals for each $63 \mathrm{~min}$ session. These values were averaged to obtain mean levels for each minute. Mean values of PLS were transformed by subtracting a constant of 0.675 from each. This served to relocate PLS scores so that the $90^{\circ}$ setting labeled 'usual' took on a value of 0.0 , and deviations from it, in the clockwise and counterclockwise directions (corresponding to increases and decreases in PLS), had maximum values \pm 0.675 , respectively.

\section{Results}

Changes in phantom limb sensations (PLS) during TENS and placebo sessions

Fig. 1 shows a minute by minute plot of mean PLS within the 7 periods for the TENS and placebo sessions. These data were submitted to a $2 \times 7$ repeated measures ANOVA using Session (TENS and placebo) as the repeated measures factors and Period (B1 through B4) as the independent sam-

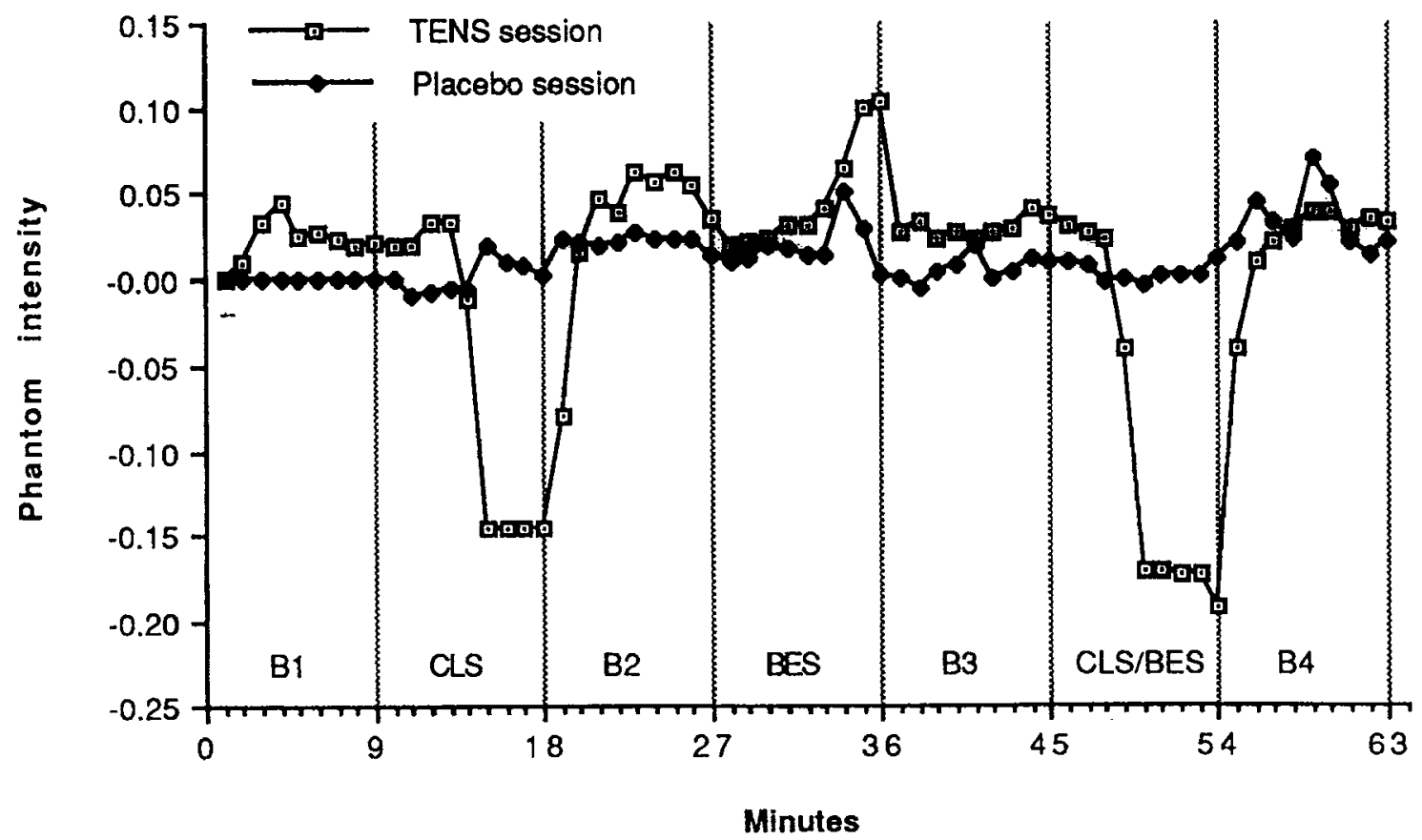

Fig. 1. Mean subjective intensity of non-painful phantom limb sensations across the 7 periods during TENS and placebo sessions. Intensity scores have been transformed so that a value of 0.0 represents the subject's normal level of phantom paresthesias. Initial resting baseline (B1), contralateral leg stimulation (CLS), first recovery baseline (B2), bilateral outer ear stimulation (BES), second recovery baseline (B3), combined CLS and BES (CLS/BES), final resting baseline (B4). 
ples factor. The analysis revealed significant effects for Period $(F(6,56)=10.55, P<0.0001)$ and for the Period $\times$ Session interaction $(F(6$, $56)=6.06, P<0.0001$ ). Simple main effects of the 2-way interaction for Period during TENS and placebo were significant $(F(6,56)=8.02, P<$ 0.0001 and $F(6,56)=14.25, P<0.0001$, respectively).

In order to explore the nature of the differences in PLS within the TENS and placebo sessions the following set of 6 mutually orthogonal contrasts were evaluated across the 7 periods in each session: contrast 1 compares phantom activity during the 4 baseline periods with that during the 3 stimulation periods. Contrasts 2, 3 and 4 compare $B 1$ with $B 2, B 3$ with $B 4$, and the joint effects of $B 1$ and B2 with that of B3 and B4, respectively. Contrast 5 compares PLS during CLS/BES with the joint effects of CLS and BES, and contrast 6 compares CLS and BES. Using Scheffé's [19] method for post hoc hypothesis testing, the first fifth and sixth contrasts reached conventional levels of significance $(P<0.05)$ for the TENS session. These results indicate that (1) during electrical stimulation PLS were significantly reduced when compared with the baseline periods, (2) mean levels of PLS did not differ significantly across the baseline periods, (3) CLS/BES resulted in significantly lower levels of PLS than did the combined effects of CLS alone and BES alone, and (4) levels of PLS were significantly higher during BES than CLS. For the placebo session, quite a different pattern of results emerged. Contrasts 2,3 , and 6 reached significance indicating differences in PLS during the 2 first and 2 last baseline periods and significantly different levels during placebo BES and CLS.

Analyses of the simple main effect of Session at each of the 7 periods revealed that mean levels of PLS were significantly lower in the TENS session than the placebo session for CLS alone and CLS/BES $(F(1,56)=8.39, P<0.01$ and $F(1$, $56)=24.6, P<0.01$ respectively), but did not differ significantly during the baseline or BES periods.

Changes in stump skin temperature (ST) during TENS and placebo sessions

Mean skin temperature of the stump across the 2 sessions is displaced in Fig. 2. The most striking

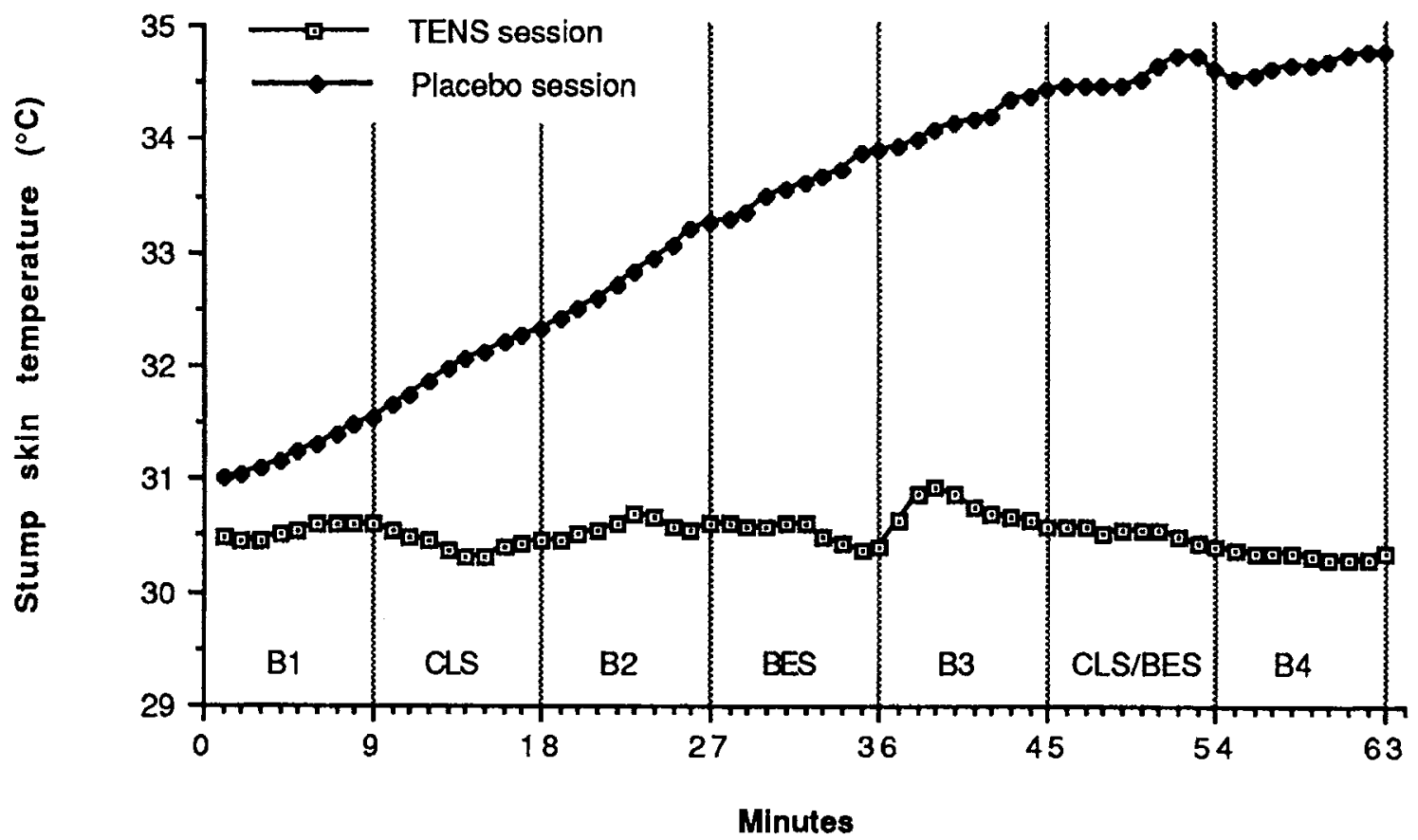

Fig. 2. Mean stump skin temperature $\left({ }^{\circ} \mathrm{C}\right)$ across the 7 periods during TENS and placebo sessions. Abbreviations as in Fig. 1. 


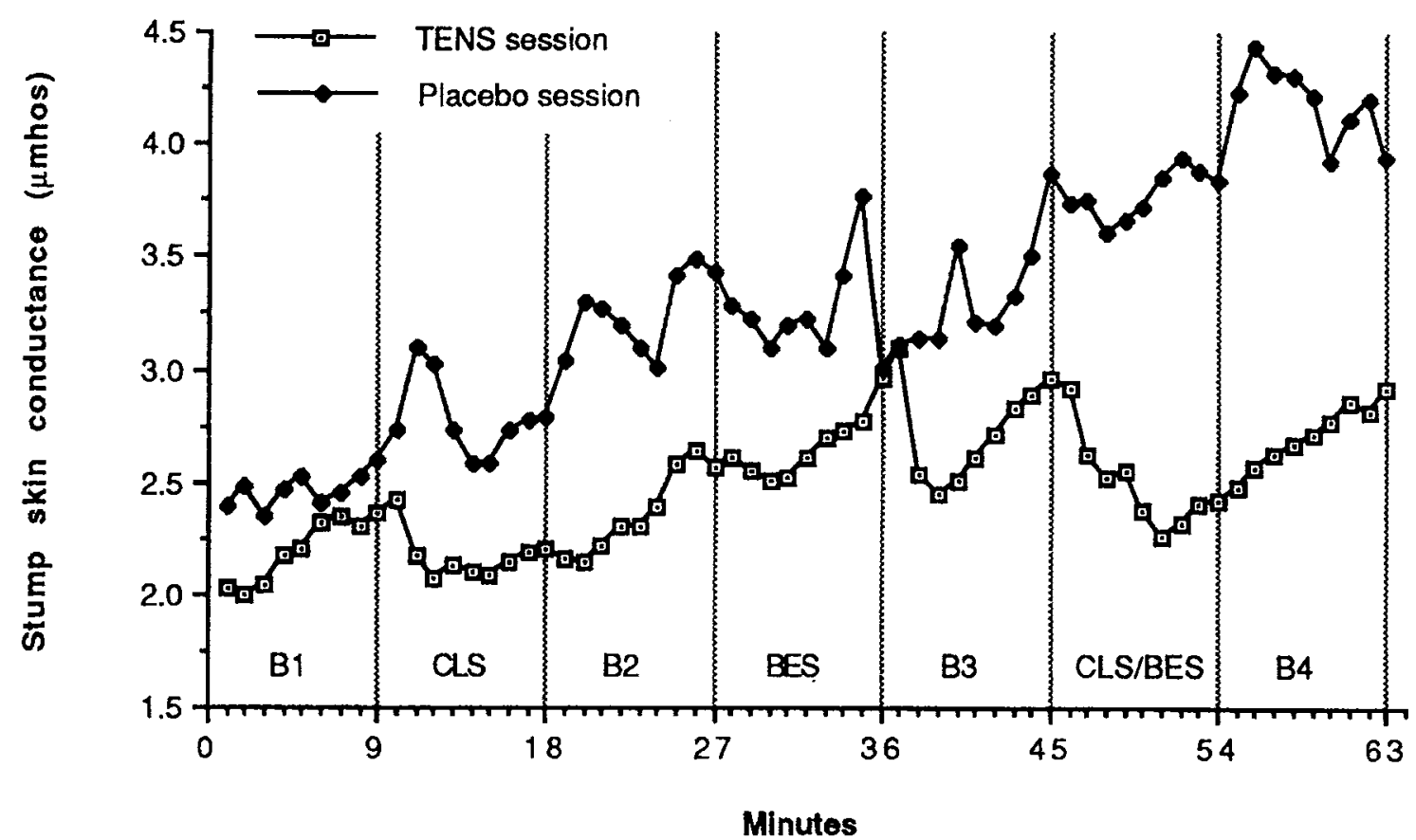

Fig. 3. Mean stump skin conductance levels ( $\mu$ mhos) across the 7 periods during TENS and placebo sessions. Abbreviations as in Fig. 1.

aspect is the anomalous increase (of $3^{\circ} \mathrm{C}$ ) from initial to final baseline periods during the placebo session. This contrasts with the more typical fluctuating pattern shown during the TENS session. Because of the obvious differences in ST during the initial baseline periods and the anomalous increase during the placebo session, we believe that further analyses of these data would be misleading. Nevertheless, they are presented for subsequent reference.

TABLE I

COEFFICIENTS OF DETERMINATION $\left(r^{2}\right)$ FOR THE STEPWISE REGRESSION ANALYSES OF STUMP SKIN CONDUCTANCE LEVELS USING LINEAR AND QUADRATIC COMPONENTS OF TIME AS PREDICTORS

\begin{tabular}{|c|c|c|c|c|c|c|c|c|}
\hline & & B1 & CLS & B2 & BES & B3 & CLS/BES & B4 \\
\hline \multirow[t]{2}{*}{$\begin{array}{l}\text { TENS } \\
\text { session }\end{array}$} & $\begin{array}{c}\text { Step } 1^{a} \\
\text { linear }\end{array}$ & $0.90^{* *}$ & 0.11 & $0.91 * *$ & $0.70 * *$ & 0.09 & $0.60^{*}$ & $0.97 * *$ \\
\hline & $\begin{array}{l}\text { Step } 2^{b} \\
\quad \text { linear }+ \\
\text { quadratic }\end{array}$ & - & $0.74 * *$ & - & $0.95 * *$ & $0.63^{*}$ & $0.91 * *$ & - \\
\hline \multirow[t]{2}{*}{$\begin{array}{l}\text { Placebo } \\
\text { session }\end{array}$} & $\begin{array}{l}\text { Step } 1^{a} \\
\text { linear }\end{array}$ & $0.43^{*}$ & 0.15 & 0.33 & 0.05 & $0.55 *$ & $0.50^{*}$ & $0.49^{*}$ \\
\hline & $\begin{array}{l}\text { Step } 2^{b} \\
\text { linear }+ \\
\text { quadratic }\end{array}$ & - & - & - & - & - & - & - \\
\hline
\end{tabular}

a $d f=(1,8)$.

b $d f=(2,8)$.

* $P<0.05 ; * * P<0.001$. 
Changes in stump skin conductance levels (SCL) during TENS and placebo sessions

Fig. 3 shows stump skin conductance levels averaged every minute across the 7 periods for the 2 sessions. Visual inspection of the data from the TENS session reveals a fairly consistent pattern of stump electrodermal activity across the baseline periods and a different but likewise consistent pattern during periods of stimulation. Stump SCL appear linear during the baseline periods and curvilinear during stimulation. This pattern is not exhibited during the placebo session.

In order to statistically describe and evaluate the pattern of electrodermal activity across periods and sessions, a separate stepwise regression analysis of SCL was performed for each period forcing the linear and quadratic components of time into the equation as predictors. Table I contains the coefficients of determination $\left(r^{2}\right)$ for the linear and quadratic equations obtained from each analysis. Table entries represent the proportion of the total variance in stump SCL accounted for by the regression equation at that step. To simplify reading in the table, values of $r^{2}$ have been omitted when the unique contribution of the quadratic component to the overall regression (over and above the linear) is not significant (i.e., $P>0.05$ ). Consequently, when reading in the table, a statistically significant linear regression indicates that a significant proportion of the total variance in $\mathrm{SCL}$ within the period under evaluation can be explained by a straight-line relationship with time, and a statistically significant quadratic regression indicates that the relationship between SCL and time is well described by a one-bend curve.

It can be seen that during the TENS session, the best description of the relationship between stump SCL and time is a straight line for the baseline periods and a positively accelerated onebend curve (see Fig. 3) for the stimulation periods. The sole exception is the B3 period but Fig. 3 shows that the main contribution to the significant quadratic regression for this period derives from the first minute which immediately followed bilateral ear stimulation (BES) and probably reflects a carry-over effect of BES into the following B3 period. For when the first minute is excluded from the analysis the coefficient of determination in- creases significantly from 0.09 to 0.90 for the linear regression. Taken together these data indicate that the onset of electrical stimulation in periods CLS, BES, and CLS/BES is associated with an initial decrease in stump SCL followed several minutes later by an increase. On the other hand, during the baseline periods SCL increase as a linear function of time and with what appears to be remarkably consistent slopes.

An examination of Fig. 3, in conjunction with the coefficients of determination in Table I, indicates that during the placebo session the behavior of stump SCL is not tied to the onset and offset of CLS, BES, and CLS/BES as it is for the TENS session. The relationship between stump SCL and time is linear for several of the placebo session periods (e.g., B1, B3, CLS/BES, B4), although none of the stimulation periods shows a significant quadratic relationship.

Relation between stump skin conductance levels (SCL) and phantom sensations (PLS)

Fig. 4 shows the relationship between stump SCL and PLS on both sessions (note that the skin conductance scale is different for Figs. 3 and 4). The Pearson correlation coefficient calculated between these variables was significantly different from zero for both the TENS $(r(61)=0.41, P<$. $0.001)$ and placebo $(r(61)=0.52, \quad P<0.0001)$ sessions. These findings indicate that in general, changes in stump SCL and PLS tend to occur in tandem, regardless of whether or not TENS is delivered. That is, changes in skin conductance correlate significantly with changes in phantom sensations both in TENS and in placebo sessions.

In an effort to determine the temporal relationship between these variables, time lag correlations were computed using the raw $(2 \mathrm{sec})$ data. For both sessions, values of skin conductance were lagged 2, 4 and $6 \mathrm{sec}$ behind values of PLS and correlation coefficients were evaluated for each lag. This procedure was repeated, lagging values of PLS behind those of SC. None of the correlations improved (even minimally) the originally significant correlation. Thus, we were unable to determine whether skin conductance responses preceded or followed changes in PLS. 
Subjective reports during monitoring of phantom limb sensations (PLS)

At the start of each session the subject was asked to describe what he felt in the phantom. Phantom sensations consisted of a 'numbness' that defined the region of his outer 3 toes. Within this relatively small area sensations were not static, but continuously changing. He described these normal changes as rapid 'waves' of numbness that increase and decrease the intensity of the involved phantom parts. Some of these 'waves' were extremely subtle while others were much more noticeable.

At the beginning of the fifth minute of contralateral leg stimulation (CLS) on the TENS session the subject spontaneously remarked 'I feel

TENS Session

A

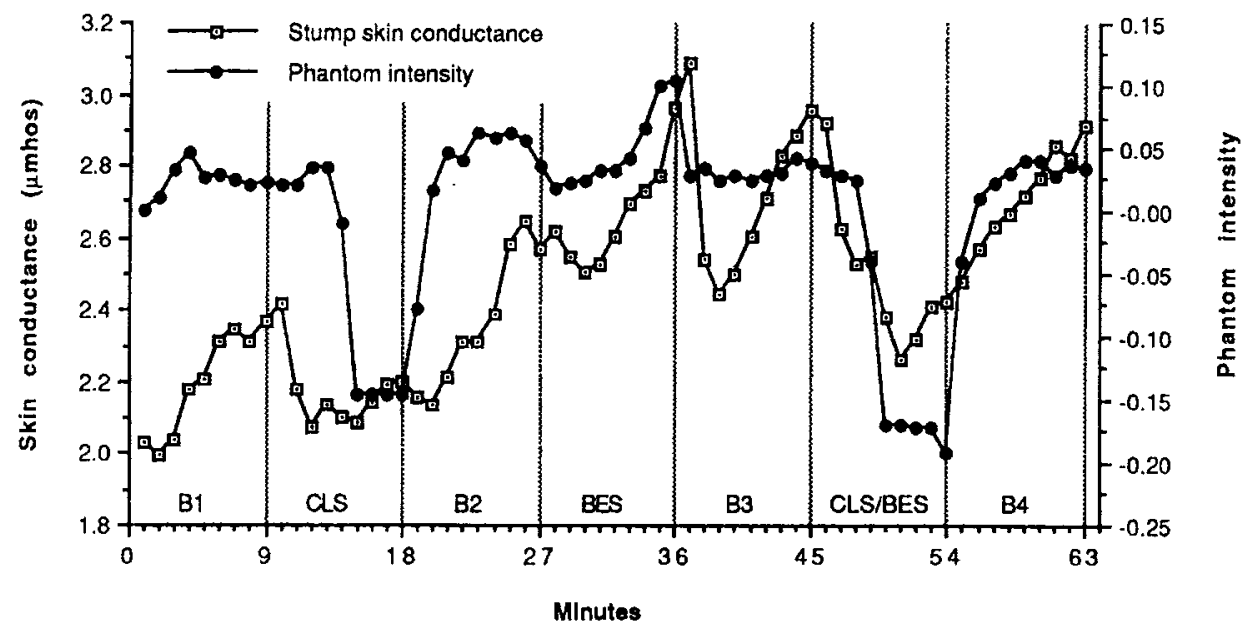

Placebo Session

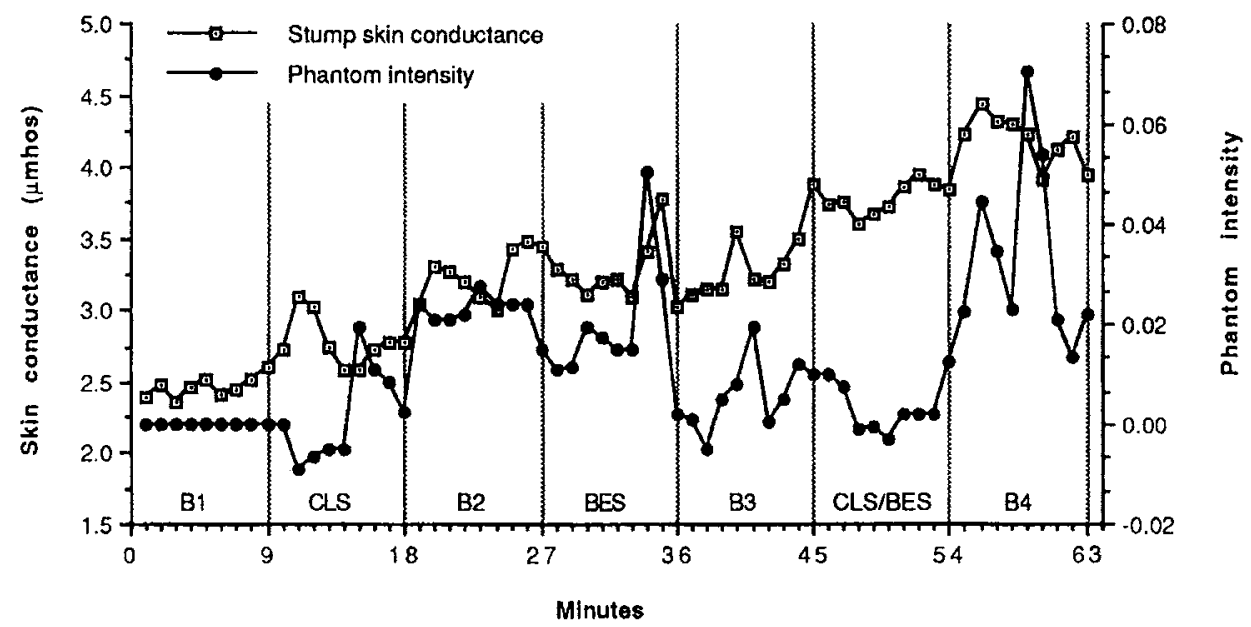

Fig. 4. Mean stump skin conductance levels and subjective intensity of non-painful phantom limb sensations across the 7 periods during TENS (A) and placebo session (B). Abbreviations as in Fig. 1. 
practically no phantom pain' * and explained that the numbness that defined his phantom toes was practically gone. Near the end of the fourth minute of CLS/BES on the same session he once again reported that the numbness in his phantom toes had practically gone and added that the phantom calf muscle was 'tired' as if he had 'walked a lot or done too much exercise." It was not unpleasant. According to the subject this phantom sensation was not in the same location as, or similar in quality to, the TENS electrodes stimulating his left calf. This 'tired' sensation was not apparent during the final resting baseline period that followed. At the end of the TENS session we were able to reproduce this sensation of tiredness with electrical stimulation applied to the contralateral leg alone (i.e., without concomitant ear stimulation).

\section{Discussion}

The results of the present case study indicate that TENS applied to the contralateral extremity was significantly more effective than a placebo in decreasing the intensity of non-painful phantom limb paresthesias, whereas electrical stimulation applied to the outer ears led to a non-significant increase. These findings are consistent with our earlier studies in which electrical stimulation applied to the outer ears failed to produce significant relief of chronic pain when compared to a control condition [15], but did lead to reports of referred sensations in a large percentage of chronic pain patients [12,15].

Whether these stimulation-induced changes in the intensity of phantom paresthesias reflect supraspinal [14], segmental [9], or both types of mechanisms cannot be answered but the results of Fitzgerald's [9] study seem particularly relevant. Fitzgerald demonstrated the existence of segmen-

\footnotetext{
* This subject, as well as other amputees we have encountered, referred indiscriminately to all phantom sensations as 'phantom pain' even when not in pain, as subsequent verification revealed. Paradoxically, this is more often true of the patients who have little or no phantom pain at all.
}

tal contralateral control over dorsal horn cells in lamina 5 of the spinal rat. Electrical stimulation of the contralateral sural nerve, which contains cutaneous afferents only, produced excitatory effects in cells with inhibitory receptive fields on the contralateral lower leg or foot. These effects were obtained using A-fiber but not $\mathrm{C}$-fiber stimulation. Inhibitory effects of contralateral A-fiber stimulation have also been observed $[4,16]$. Together, these studies may shed some light on the following finding. During TENS applied to the contralateral calf, our subject reported an awareness of his phantom calf while at the same time noticing a decrease in the intensity of the paresthesias in his phantom toes. We assume that under normal circumstances the subject's perception of his phantom toes results from the lack of inhibitory control exerted over spinal cord cells subserving these regions, and the absence of his phantom calf is evidence of the redevelopment of this control. The effect of contralateral leg stimulation on spinal cord cells subserving the phantom might be to facilitate inhibitory control in regions lacking it and disinhibit those for which it had already redeveloped. This would explain the simultaneous but opposite effects the subject reported in the intensity of his phantom toes and calf.

The finding of a significant direct relationship between stump skin conductance levels and the intensity of phantom limb sensations is support for an association between sympathetic activity and paresthesias referred to the phantom. In the absence of evidence on the temporal relation between these 2 variables the nature of their association remains obscure. Nevertheless we suggest 2 possible hypotheses to account for this relationship.

The first is a more parsimonious account of the data and assumes that stump skin conductance levels and the intensity of phantom limb sensations are related to one another in the same way as a verbal acknowledgement of them is to their occurrence. According to this hypothesis the subject's detection of a change in phantom sensations produces heightened electrodermal activity in the form of a skin conductance response, much as any anticipated and salient signal during a vigilance 
task results in a generalized orienting response [20]. Excluding spontaneous skin conductance responses which, by definition, should be randomly distributed over time, extended intervals in which the phantom remains relatively stable at reduced intensity (e.g., CLS and CLS/BES of the TENS session) would allow levels of electrodermal activity to decrease. Thus, the overall pattern of a positive relationship between stump SCL and PLS would emerge.

A second possibility is that stump skin conductance levels correlate with the intensity of phantom limb sensations because they both reflect the activity of cholinergic postganglionic sympathetic fibers on primary afferents located in the stump and stump neuromas. Bini et al. [3] recorded multiunit sympathetic activity from human nerve fascicles and found that spontaneous activity in postganglionic sympathetic fibers to the smooth muscle of blood vessels and sweat glands is frequently followed by vasomotor and sudomotor activity in the form of vasoconstriction and electrodermal responses. Although sympathetic activity in one type of fiber (e.g., vasomotor) was often accompanied by activity in the other (i.e., sudomotor), this relation is not a necessary one and both complementary and opposite responses were recorded.

Our findings seem to reflect this potential for independent sympathetic vaso- and sudomotor activity as evidenced by the apparent dissociation of electrodermal activity and skin temperature on the placebo session. We observed a significant correlation between phantom sensations and skin conductance levels on both sessions, whereas skin temperature appeared to covary with phantom sensations (and inversely with skin conductance) only on the session in which TENS was given. It is possible that a more sensitive measure of stump blood flow might have shown the latter relationship on the placebo session as well. Nevertheless, our findings implicate the cholinergic component of the postganglionic sympathetic outflow as a possible mediator of the relationship between stump skin conductance and phantom sensations. Very little has been done in the field of pain and cholinergic chemosensitivity (see Nathan [17] and Ochoa et al. [18]), but a study by Diamond [6] showed that regenerating nerves are abnormally sensitive to the effects of acetylcholine and discharge rapidly in its presence.

According to this hypothesis, spontaneous bursts of activity in sudomotor sympathetic fibers located in the stump result in sweat gland activity and heightened skin conductance levels. At the same time, or shortly after, afferent fibers in stump neuromas would increase their rate of firing due to the liberation of acetylcholine. These impulses are referred to certain regions of the phantom as increases in paresthesias. Thus, the moment-tomoment fluctuations in the intensity of phantom limb sensations (described as 'waves' by our subject) may in part represent a sympathetic efferent-somatic afferent cycle. Increases in the intensity of phantom limb sensations would follow bursts of sympathetic activity due to neurotransmitter release and decreases would correspond to periods of relative sympathetic inactivity.

We would like to stress the finding that skin conductance levels at the stump correlated significantly with phantom limb sensations even on the placebo session. This implies that the relationship between skin conductance and phantom intensity cannot be due to TENS since TENS was not delivered on the placebo session, but that when TENS is applied contralaterally, both stump skin conductance and phantom intensity decrease. Although stump skin conductance began to drop by the first minute after CLS and CLS/BES, it was several minutes before the subject noticed and reported a change in the intensity of phantom sensations (Fig. 4A). These lags did not appear to be the result of a lapse in the subject's concentration due to the distracting influence of TENS onset, for similar lags were not evident during the same periods on the placebo session when the subject was equally distracted (Fig. 4B). Furthermore, this subject, and others tested in a similar manner (unpublished), showed that they are quite capable of continued monitoring of phantom sensations during TENS (and placebo) onset by shifting their attention from the phantom to the site of the TENS electrodes, and back again.

It is difficult to account for the concomitant changes in electrodermal activity and phantom sensations from TENS to baseline periods. The 
pattern of skin conductance was consistently linear during the baseline periods indicating a progressive increase in sympathetic sudomotor activity. In contrast, it was consistently curvilinear during periods of electrical stimulation indicating an initial decrease followed by an increase in sudomotor responses. Correlates of peripheral autonomic activity during TENS have been reported but their findings are inconsistent $[1,2,7,8]$. Nevertheless, it is possible that TENS applied to the contralateral limb and outer ears leads to an initial reduction in sympathetic sudomotor activity in the stump and surrounding tissue which decreases primary afferent discharge and leads to reduced phantom sensations. Further research is underway to examine some of the possibilities raised by the present study.

\section{Acknowledgements}

We would like to thank Dr. Blaine Ditto for the use of his psychophysiology laboratory, Ms. Rhonda Amsel for her advice on statistical matters and Dr. Peter Nathan for his helpful comments on an earlier draft of the manuscript.

This research was supported by Medical Research Council of Canada Studentships to J.K. and C.F. and by Grant A7891 from the Natural Sciences and Engineering Research Council of Canada to R.M.

\section{References}

1 Abram, S.E., Increased sympathetic tone associated with transcutaneous electrical stimulation, Anesthesiology, 45 (1976) 575-577.

2 Abram, S.E., Asiddao, C.B. and Reynolds, A.C., Increased skin temperature during transcutaneous electrical stimulation, Anesth. Analg., 59 (1980) 22-25.

3 Bini, G., Hagbarth, K.E., Hynninen, P. and Wallin, B.G., Thermoregulatory and rhythm-generating mechanisms governing the sudomotor and vasoconstrictor outflow in human cutaneous nerves, J. Physiol. (Lond.), 306 (1980) 537-552.

4 Brown, A.G., Kirk, E.J. and Martin, H.G., Descending and segmental inhibition of transmission through the spinocervical tract, J. Physiol. (Lond.), 230 (1973) 689-705.

5 Carabelli, R.A. and Kellerman, W.C., Phantom limb pain: relief by application of TENS to contralateral extremity, Arch. Phys. Med. Rehab., 66 (1985) 466-467.

6 Diamond, J., The effect of injecting acetylcholine into normal and regenerating nerves, J. Physiol. (Lond.), 145 (1959) 611-629.

7 Dooley, D.M. and Kasprak, M., Modification of blood flow to the extremities by electrical stimulation of the nervous system, Sth. Med. J. (Bgham, AL), 69 (1976) 1309-1311.

8 Ebersold, M.J., Laws, E.R. and Albers, J.W., Measurements of autonomic function before, during and after transcutaneous stimulation in patients with chronic pain and in control subjects, Mayo Clin. Proc., 52 (1977) 228-232.

9 Fitzgerald, M., The contralateral input to the dorsal horn of the spinal cord in the decerebrate spinal rat, Brain Res., 236 (1982) 275-287.

10 Fowles, D.C., Christie, M.J., Edelberg, R., Grings, W.W., Lykken, D.T. and Venables, P.H., Publication recommendations for electrodermal measurements, Psychophysiology, 18 (1981) 232-239.

11 Gross, D., Contralateral local anaesthesia in the treatment of phantom limb and stump pain, Pain, 13 (1982) 313-320.

$12 \mathrm{Katz}$, J. and Meizack, R., Somatosensory memory for pain after amputation, Can. Psychol., 29 (1988) abstr. no. 407.

$13 \mathrm{Katz}$, J. and Melzack, R., Referred sensations in chronic pain patients, Pain, 28 (1987) 51-59.

14 Le Bars, D., Dickenson, A.H. and Besson, J.H., Diffuse noxious inhibitory controls (DNIC). II. Lack of effect on non-convergent neurons, supraspinal involvement and theoretical implications, Pain, 6 (1979) 305-327.

15 Melzack, R. and Katz, J., Auriculotherapy fails to relieve chronic pain: a controlled crossover study, JAMA, 251 (1984) 1041-1043.

16 Mendell, L.M., Physiological properties of unmyelinated fibre projection to the spinal cord, Exp. Neurol., 16 (1966) 316-332.

17 Nathan, P.W., Involvement of the sympathetic nervous system in pain. In: H.W. Kosterlitz and L.Y. Terenius (Eds.), Pain and Society, Verlag Chemie, Weinheim, 1980, pp. 311-324.

18 Ochoa, J.L., Torebjörk, E., Marchettini, P. and Sivak, M., Mechanisms of neuropathic pain: cumulative observations, new experiments, and further speculation. In: H.L. Fields, R. Dubner and F. Cervero (Eds.), Advances in Pain Research and Therapy, Vol. 9, Raven Press, New York, 1985, pp. 431-450.

19 Scheffe, H., A method for judging all contrasts in the analysis of variance, Biometrika, 40 (1953) 87-104.

20 Voronin, L.G., Bonfitto, M. and Vasileva, V.M., The interrelation of the orienting reaction and conditioned reflex to time in man. In: E.N. Sokolov and O.S. Vinogradova (Eds.), Neuronal Mechanisms of the Orienting Reflex, Erlbaum, Englewood Cliffs, NJ, 1975, pp. 252-263.

21 Wall, P.D., On the origin of pain associated with amputation. In: J. Siegfried and M. Zimmermann (Eds.), Phantom and Stump Pain, Springer, Berlin, 1981, pp. 2-14.

22 Winnem, M.F, and Amundsen, T., Treatment of phantom limb pain with TENS, Pain, 12 (1982) 299-300. 